\title{
Combining Crop Growth Modeling With Trait-Assisted Prediction Improved the Prediction of Genotype by Environment Interactions
}

\author{
Pauline Robert, Jacques Le Gouis, The BreedWheat Consortium and Renaud Rincent* \\ INRAE, UCA, UMR 1095 Génétique, Diversité et Ecophysiologie des Céréales, Clermont-Ferrand, France
}

Plant breeders evaluate their selection candidates in multi-environment trials to estimate their performance in contrasted environments. The number of genotype/environment combinations that can be evaluated is strongly constrained by phenotyping costs and by the necessity to limit the evaluation to a few years. Genomic prediction models taking the genotype by environment interactions (GEI) into account can help breeders identify combination of (possibly unphenotyped) genotypes and target environments optimizing the traits under selection. We propose a new prediction approach in which a secondary

OPEN ACCESS

Edited by: Rodomiro Ortiz,

Swedish University of Agricultural Sciences, Sweden

Reviewed by:

Jesse Poland,

Kansas State University, United States Sebastian Michel,

University of Natural Resources and Life Sciences, Vienna, Austria

Hans-Peter Piepho,

University of Hohenheim, Germany

*Correspondence:

Renaud Rincent

renaud.rincent@inrae.fr

Specialty section:

This article was submitted to Plant Breeding,

a section of the journal

Frontiers in Plant Science

Received: 28 January 2020

Accepted: 22 May 2020

Published: 19 June 2020

Citation:

Robert P, Le Gouis J and

Rincent $R$ (2020) Combining Crop

Growth Modeling With Trait-Assisted

Prediction Improved the Prediction

of Genotype by Environment

Interactions. Front. Plant Sci. 11:827.

doi: 10.3389/fp/s.2020.00827 trait available on both the calibration and the test sets is introduced as an environment specific covariate in the prediction model (trait-assisted prediction, TAP). The originality of this approach is that the phenotyping of the test set for the secondary trait is replaced by crop-growth model (CGM) predictions. So there is no need to sow and phenotype the test set in each environment which is a clear advantage over the classical traitassisted prediction models. The interest of this approach, called CGM-TAP, is highest if the secondary trait is easy to predict with CGM and strongly related to the target trait in each environment (and thus capturing GEI). We tested CGM-TAP on bread wheat with heading date as secondary trait and grain yield as target trait. Simple CGMTAP model with a linear effect of heading date resulted in high predictive abilities in three prediction scenarios (sparse testing, or prediction of new genotypes or of new environments). It increased predictive abilities of all reference GEl models, even those involving sophisticated environmental covariates. Keywords: crop growth model, gene-based modeling, genomic selection, genotype $\mathrm{x}$ environment interaction,
multi-environment trials, wheat

\section{INTRODUCTION}

The objective of plant breeders is to develop varieties well adapted to target environments. For this purpose, they evaluate each year candidate varieties in multi-environment trials (MET). Given that the speed of the process is a key element of genetic progress and since phenotyping is expensive, most varieties are evaluated in a small number of environments, considered as a combination of year $\times$ site $\times$ condition. Consequently, the environments in which the varieties are evaluated can be quite different from the target environments, because of the significant variation between years. In addition, only a limited number of varieties are evaluated each year to control the phenotyping costs. All these constraints are reducing the chance of success as they limit the number of lines that can be evaluated in the target environments. 
One way to raise this strong constraint is to predict the performance of candidate varieties using molecular information with genomic selection (GS) models (Whittaker et al., 2000; Meuwissen et al., 2001). In GS, a phenotyped and genotyped calibration set is used to estimate DNA marker effects. Once the model is calibrated, new candidate varieties can be predicted, as long as their genotypic information is available. Specific GS models were proposed to predict the performance of varieties in different environments, taking the genotype by environment interaction (GEI) into account. It was first proposed to adapt the reference GS models to the GEI context by attributing environment specific effects to the markers (Schulz-Streeck et al., 2013; Crossa et al., 2016), or by modeling genetic covariances between environments (Burgueño et al., 2012). In other studies, environmental covariates (EC) were introduced in the GS model (Heslot et al., 2014; Jarquín et al., 2014; Malosetti et al., 2016; Ly et al., 2018), which allows predicting the performance of varieties in new environments. Crop growth models (CGM) were sometimes used to adjust the EC estimates to phenological stages, or to derive EC estimating the stress experienced by the plants (Ly et al., 2017; Rincent et al., 2019), instead of directly using pedoclimatic data.

One efficient way to improve the prediction accuracy of GEI is to introduce secondary traits measured in each environment on both the calibration set and the test set in multi-trait GS models. It was indeed shown that if the secondary trait is sufficiently heritable and correlated to the target trait, the approach called "trait-assisted prediction" (TAP) or "phenotype imputation", can be very efficient (Henderson and Quaas, 1976; Calus and Veerkamp, 2011; Jia and Jannink, 2012; Pszczola et al., 2013; Fernandes et al., 2018; Bustos-Korts et al., 2019). It resulted in successful applications in wheat (Rutkoski et al., 2016; Sun et al., 2017, 2019; Crain et al., 2018; Lado et al., 2018; Michel et al., 2018; Schulthess et al., 2018). In particular Rutkoski et al. (2016) showed that secondary physiological traits measured in each environment on both predicted and test sets resulted in increased predictive ability for grain yield using a TAP model. TAP is particularly useful when the secondary trait is easy to obtain, highly heritable and strongly correlated to the target trait. In MET, the secondary trait measured in each environment can capture GEI and serves as a proxy of the target trait in each environment (Bustos-Korts et al., 2019). But the applicability of this approach in the GEI context is considerably constrained by the necessity to phenotype all varieties (calibration and test sets) in all environments for the secondary trait, which means that the test set has to be sown and phenotyped in each environment. The interest of this approach would be considerably stronger if the secondary trait would be accessible without phenotyping the test set.

Crop Growth Models (CGM) are also powerful tools to predict GEI. They model plant development using mechanistic relationships with genetic characteristics (genetic parameters) and environmental variables as input. These genetic parameters characterize the varieties (e.g., sensitivity to photoperiod) independently from the environment, and so are supposed to be constant from one environment to another for a given variety (Reymond et al., 2003, 2004). A method combining CGM and GS, called gene-based modeling (GBM), can be used to predict GEI for unobserved varieties. If the genetic parameters of varieties of the calibration set are known, a GS model can be calibrated and used to predict the genetic parameters of the test varieties. These predictions can then be used as input for the CGM together with environmental variables to predict the performance of the test set in various environments.

This strategy has proven to be effective on simple traits such as leaf elongation rate in maize (Reymond et al., 2003; Chenu et al., 2008), fruit quality (Quilot et al., 2005; Prudent et al., 2011), and phenology of various species (White and Hoogenboom, 1996; Nakagawa et al., 2005; Yin, 2005; Messina et al., 2006; White et al., 2008; Uptmoor et al., 2011; Zheng et al., 2013; Onogi et al., 2016) including wheat (Bogard et al., 2014; Rincent et al., 2017). The major limit of this approach is that it remains difficult to efficiently predict complex traits such as yield, although promising results have been obtained (Technow et al., 2015; Cooper et al., 2016; Messina et al., 2018). In any case, GBM can be very efficient to predict secondary traits, for example related to phenology. If we were able to use CGM (or GBM for new varieties) to accurately predict a secondary trait correlated to the final target trait in each environment, then we would be able to combine CGM and trait assisted prediction to overcome the limits of both approaches. The idea here is to use a TAP approach in which the secondary trait is not phenotyped for the test set (so there is no need to sow the test set in any experiment) but predicted in each environment of interest thanks to CGM.

We propose to test this new approach (CGM-TAP) on winter wheat with grain yield (GY) as target trait (the trait the breeder is interested in), and heading date (HD) as secondary trait. HD is very highly correlated to flowering time, which is indeed an important adaptive trait with an optimal date depending on the environment. Phenology is a major trait for the plants to benefit from the most possible resources while avoiding stressing conditions at key stages (Richards, 1991; Lopes et al., 2014; Semenov et al., 2014; Flohr et al., 2017). In addition, it was shown that GBM was efficient to predict HD in winter wheat (Bogard et al., 2014; Rincent et al., 2017). Therefore, this trait seems to be a particularly good candidate to test the CGM-TAP approach with GY as target trait. For this purpose, we used a MET composed of 220 varieties and 42 managed environments, that was used to compare the accuracy of CGM-TAP to reference GEI models in different prediction scenarios: the prediction of new varieties, of new environments, or of the missing phenotypes of an incomplete design (sparse testing).

\section{MATERIALS AND METHODS}

\section{Genetic Material, Genotyping, and Estimation of a Genetic Covariance Matrix (Kinship)}

The plant material has been previously described in Rincent et al. (2018, 2019) and Touzy et al. (2019). The genetic panel is composed of 220 European elite varieties of winter wheat. It was genotyped with the TaBW280K high-throughput genotyping 
array described in Rimbert et al. (2018). This array was designed to cover both genic and intergenic regions of the three bread wheat subgenomes. Markers with a minor allele frequency (MAF) below $5 \%$, or with heterozygosity or missing data rate above $5 \%$ were removed. Markers in strong Linkage Disequilibrium (LD) were filtered out using the pruning function of Plink (Purcell et al., 2007) with a window of size 100 SNPs, a step of 5 SNPs and a LD threshold of 0.8, as proposed in Charmet et al. (2020). Eventually, we obtained 26,116 polymorphic high resolution SNPs, with an average missing data rate of $1.0 \%$. Missing values were imputed as the marker observed frequency.

Genotype of individual $i$ at marker $l\left(M_{i, l}\right)$ was coded as $1,0.5$, or 0 for homozygote for an arbitrarily chosen allele, heterozygote, and the other homozygote, respectively. Genomic relatedness (kinship) between individuals was estimated following (VanRaden, 2008):

$$
K_{i, j}=\sum_{l=1}^{L} \frac{\left(M_{i, l}-p_{l}\right)\left(M_{j, l}-p_{l}\right)}{\mathrm{b}}, \text { with } b=\sum_{l=1}^{L} p_{l} \times\left(1-p_{l}\right), p_{l} \text { being }
$$

the allelic frequency of the reference allele in the corresponding diversity panel, $L$ the number of markers. The matrix of genomic relatedness coefficients will be denoted $K$ in the manuscript.

\section{Phenotypic Data Measured in the Multi-Environment Trial}

The same phenotypes as in Rincent et al. (2019) were used. Briefly, the panel was phenotyped for grain yield (GY) and heading date (HD) in a multi-environment trial composed of 42 managed environments located in France between 2012 and 2016 (Supplementary Table S1). These 42 managed environments correspond to 26 combinations of years and locations, with two treatments for 16 of them. Among these 16 combinations of years and locations, three had an irrigated (WW) and a rainfed (WD) treatment, one had a well-watered (WW) and a rainout shelter (RO) treatments, and 12 had a high (HN) and a low (LN) nitrogen fertilizer treatments. These 42 combinations of location, year and management will be called environments in the rest of the document. Experimental designs, estimation of adjusted means and heritabilities were presented in Rincent et al. (2019). Briefly summarized, in each environment the varieties were grouped in six to eight blocks according to their earliness. The designs were two-replicate designs or augmented designs. Adjusted means and heritabilities were estimated with SpATS (Rodríguez-Álvarez et al., 2018) for the two-replicate designs to take spatial trends into account and with block effect only for the augmented designs. On this dataset, the use of weights in the second step of the analysis to take into account the difference of precision of the adjusted means (Damesa et al., 2017) did not improve the results, and so a basic analysis without weights was applied here.

\section{Environmental Characterization and Estimation of an Environmental Covariance Matrix}

Each environment was characterized by 139 environmental covariates (EC) in Rincent et al., 2019. Seventy-two of these covariates were estimated using climatic data (temperature, radiation), and 67 were estimated with SiriusQuality CGM (Martre et al., 2006) as dry matter stress index. These 139 covariates were estimated in each environment, transformed to standard normal distributions, and compiled in a matrix $\Omega$ of dimension $(42 \times 139)$.

$\Omega$ was then used to estimate a covariance matrix between environments (W). We applied the approach of Jarquín et al. (2014) in which all the ECs were used to estimate the covariance matrix. Environments with similar stresses are assumed to have similar GxE patterns. To compute $W$, we proceeded in two steps: first we computed the Euclidean distance matrix between environments $\left(D_{\Omega}\right)$ with the matrix of environmental covariates $(\Omega)$, and then the covariance matrix $W$ was computed as: $W=$ $1_{E}-\frac{D_{\Omega}}{\max \left(D_{\Omega}\right)}$. W does not take into account the targeted response variable, but the EC are supposed to be helpful to give an estimate of the true unknown environmental covariance matrix, as they reflect the conditions experienced by the plants. $W$ was used in the downstream analysis as an estimate of the environmental covariance matrix.

\section{Selection of a Subset of Representative Environments}

Because of the redundancy in the dataset due to strong similarities between the environments (Rincent et al., 2019), and to limit computational burden, a subset of sixteen environments was selected. The selection was applied in such a way that the different kinds of HD/GY relationship were explored. The 16 environments were composed of four environments with a low linear correlation between HD and GY (absolute value of the correlation $<0.3$ ), four environments with an intermediate $\mathrm{HD} / \mathrm{GY}$ correlation (absolute value of the correlation between 0.3 and 0.6), four environments with a strong HD/GY correlation (absolute value of the correlation above 0.6), and four environments for which a quadratic relationship explained more than a linear relationship (Table 1). These four groups of environments were named "low," "medium," "high," and "quadratic" according to the kind of relationship between HD and GY. The 16 environments were randomly sampled among the 42 environments of Rincent et al. (2019) with the constraint that each of the four groups of environments were represented by four environments. The prediction scenarios presented below were applied to these sixteen environments.

\section{Prediction Objectives and the Corresponding Cross-Validation Schemes}

Three prediction objectives were considered: the prediction of observed varieties in observed environments (oGoE) or in new environments (oGnE), and the prediction of new varieties in observed environments $(\mathrm{nGoE})$. oGoE consists of predicting missing values in a MET (sparse testing), which typically corresponds to the situation faced by breeders when some observations are missing in their trial networks. oGnE and $\mathrm{nGoE}$ are more ambitious because predictions are made in an environment or for a variety without any phenotypic information on it. 
TABLE 1 | Description of the 16 environments selected for the analysis.

\begin{tabular}{llcrrr}
\hline Environment & Group $^{\mathbf{a}}$ & Correlation HD/GY & $\hat{\alpha}^{\mathbf{b}}$ & $\hat{\boldsymbol{\alpha}}^{\mathbf{c}}$ & $\hat{\boldsymbol{\beta}}^{\mathbf{d}}$ \\
\hline Rea13WW & Low & -0.06 & -0.34 & -0.33 & -0.32 \\
Cle13LN & Low & 0.19 & 0.14 & 0.10 & -0.20 \\
Sau13HN & Low & 0.25 & 0.17 & 0.17 & 0.01 \\
Gre12WW & Low & -0.28 & -0.64 & -0.64 & -0.39 \\
Mon12LN & Medium & 0.50 & 0.81 & 0.83 & -0.27 \\
Cap12LN & Medium & 0.51 & 0.59 & 0.68 & -1.03 \\
Gre13WD & Medium & -0.54 & -0.82 & -0.82 & 0.01 \\
Mon13LN & Medium & 0.59 & 1.03 & 1.03 & -0.19 \\
Vra13LN & High & 0.61 & 0.73 & 0.74 & 0.06 \\
Gre14WD & High & -0.64 & -0.81 & -0.82 & -0.90 \\
Gre12WD & High & -0.68 & -0.99 & -0.98 & -0.39 \\
Rec13LN & High & 0.76 & 1.91 & 1.95 & 0.64 \\
Cap12HN & Quadratic & 0.38 & 0.18 & 0.36 & -1.56 \\
Coi12WW & Quadratic & 0.34 & 0.50 & 0.18 & -1.58 \\
Lou12LN & Quadratic & 0.35 & 0.82 & 0.39 & -1.91 \\
All14LN & Quadratic & 0.19 & 0.20 & 0.19 & -2.20 \\
\hline
\end{tabular}

"The sixteen environments were grouped in four classes according to the kind of relationship between HD and GY: "low," "medium," or "high" correlation, or "quadratic" relationship. ${ }^{b} \alpha$ estimates in the 16 environments using model EG_HD. ${ }^{c} \alpha$ and ${ }^{d} \beta$ estimates in the 16 environments using model EG_HD ${ }^{2}$. $\alpha$ and $\beta$ correspond to the coefficients for the linear and quadratic regressions of GY on $H D$ in each environment. The estimates of $\alpha$ and $\beta$ presented here were obtained with the full dataset.

To evaluate the performance of the prediction models in these three situations, three cross-validation schemes were defined: CVrandom, CVnewG, and CVnewE.

Observed varieties in observed environments was addressed by the CVrandom scheme, that is a 6-folds cross-validation, in which the folds were randomly sampled from the dataset. oGnE was addressed by the CVnewE scheme, which is a leaveone environment-out scheme, in which a new environment is predicted. nGoE was addressed by the CVnewG scheme, with a division in six folds consisting in five randomly selected groups of varieties.

Predictive abilities were computed for each fold and each environment as the correlation between adjusted means and predictions in the test set. For each cross-validation scheme except CVnewE (leave-one-out scheme), the total procedure was repeated 10 times to get robust estimates of predictive abilities.

\section{Reference Prediction Models}

Three reference models with various levels of complexity were used to predict GY in the MET. In the first kind of models, the kinship matrix allowed sharing information between varieties, but there was no sharing of information between environments:

$$
\begin{aligned}
\text { EG }: Y_{i j}=\mu_{j}+G_{i}+\epsilon_{i j}, \text { with } G_{i} \sim N\left(0, K \sigma_{g 1}^{2}\right) \\
\text { EG_GxE : } Y_{i j}=\mu_{j}+G_{i}+G E_{i j}+\epsilon_{i j}, \\
\text { with } G E_{i j} \sim N\left(0, K \otimes I_{N_{E}} \sigma_{g 2}^{2}\right)
\end{aligned}
$$

$I_{N_{E}}$ is an identity matrix of size the number of environments and $\otimes$ is the Kronecker product. $\mu_{j}$ is a fixed environmental effect, $G_{i}$ is a random polygenic effect for variety $i$, and $G E_{i j}$ is a random effect corresponding to the interaction between variety $i$ and environment $j$. In all models, $\epsilon_{i j} \sim N\left(0, I \sigma_{e}^{2}\right)$, is the residual effect. In model EG the prediction of a variety is the same for any environment (main effect model). In model EG_GxE, GEI are taken into account, but there is no sharing of information between environments. Consequently, this second model is only applicable to scenarios CVrandom and CVnewG.

In the third model, information between environments is shared thanks to an environmental covariance matrix estimated with the environmental covariates as proposed by Jarquín et al. (2014):

$$
\begin{aligned}
\text { EG_GxW }: Y_{i j}=\mu_{j} & +G_{i}+G E_{i j}+\epsilon_{i j}, \\
& \text { with } G E_{i j} \sim N\left(0, K \otimes W \sigma_{g 3}^{2}\right)
\end{aligned}
$$

$\mathrm{W}$ was estimated as described above.

\section{The Crop-Growth Modeling - Trait Assisted Prediction Model (CGM-TAP)}

Crop-growth modeling - trait assisted prediction is a trait assisted prediction model in which the secondary trait is observed for the calibration set but predicted for the test set using crop growth modelling (CGM) or gene-based crop-growth modelling (GBM) (Figure 1). The idea is to combine CGM and TAP to overcome the limit of GBM (not applicable yet to complex traits such as yield) and TAP (requires the phenotyping of the test set for the secondary trait in each environment). First (step 1), the CGM or GBM approach is applied to predict the secondary trait (HD) for

\section{Calibration set}

Test set

Target trait

Observed

???

Secondary trait

Observed

Predicted with CGM

FIGURE 1 | Schematic representation of CGM-TAP. CGM-TAP is a trait-assisted prediction approach in which the secondary trait is predicted using crop-growth modeling instead of being phenotyped. 
the test set in each environment. Then (step 2), a trait-assisted prediction model is applied to the full dataset with the secondary trait as covariate (observed for the calibration set, predicted with CGM or GBM for the test set) with an environment specific effect to predict the target trait (GY) in each environment. In more details, CGM-TAP consists in the following two steps:

- Step 1: Prediction of the secondary trait (HD) for the test set using CGM. In this first step, the two main genetic parameters of the CGM involved in phenology (sensitivity to photoperiod "SLDL" and phyllochron "P") are estimated (CVnewE and CVrandom) or predicted (CVnewG) for the test set. These variety specific genetic parameters are then used as input to the CGM together with the daily climatic data (temperature, daylength) to predict HD for the test varieties in each environment. In scenarios CVnewE and CVrandom, the test varieties are scored for HD in some environments, and so a Bayesian algorithm (Rincent et al., 2017) was used to directly estimate the genetic parameters of the test varieties. Briefly, the MCMC algorithm is a hybrid Gibbs sampler that updates the coordinates of the different parameters through a Metropolis-Hastings step using as proposal a Gaussian distribution centered on the previous value of the chain. In scenario CVnewG, there is no phenotypic information on the test varieties and so the genetic parameters cannot be estimated. So, a GBM approach was applied in which the genetic parameters are estimated for the calibration varieties, and then a GBLUP model (with major phenology markers as fixed effects) was used to predict the genetic parameters of the test varieties.

- Step 2: Different trait-assisted prediction models were then used to predict the target trait (GY) for the test set:

$$
\begin{gathered}
\text { EG_HD : } Y_{i j}=\mu_{j}+\alpha_{j} \times h_{i j}+G_{i}+\epsilon_{i j}, \\
\text { with } G_{i} \sim N\left(0, K \sigma_{g 4}^{2}\right) \\
\text { EG_GxE_HD : } Y_{i j}=\mu_{j}+\alpha_{j} \times h_{i j}+G_{i}+G E_{i j}+\epsilon_{i j}, \\
\text { with } G E_{i j} \sim N\left(0, K \otimes I_{N_{E}} \sigma_{g 5}^{2}\right) \\
\text { EG_GxW_HD : } Y_{i j}=\mu_{j}+\alpha_{j} \times h_{i j}+G_{i}+G E_{i j}+\epsilon_{i j}, \\
\text { with } G E_{i j} \sim N\left(0, K \otimes W \sigma_{g 6}^{2}\right)
\end{gathered}
$$

with $h_{i j}$ the heading date of variety $i$ in environment $j . h_{i j}$ was observed for the calibration varieties and predicted for the test set. $\alpha_{j}$ is the effect of HD on GY in environment $j$.

Three other models similar to models $4-6$ but with a quadratic effect of HD were also used:

$$
\begin{array}{r}
\text { EG_HD }{ }^{2}: Y_{i j}=\mu_{j}+\alpha_{j} \times h_{i j}+\beta_{j} \times h_{i j}^{2}+G_{i}+\varepsilon_{i j} \\
\text { EG_GxE_HD }: Y_{i j}=\mu_{j}+\alpha_{j} \times h_{i j}+\beta_{j} \times h_{i j}^{2}+G_{i}+G E_{i j}+\varepsilon_{i j}
\end{array}
$$

EG_GxW_HD ${ }^{2}: Y_{i j}=\mu_{j}+\alpha_{j} \times h_{i j}+\beta_{j} \times h_{i j}^{2}+G_{i}+G E_{i j}+\varepsilon_{i j}$ with $h_{i j}^{2}$ the squared heading date (centered and scaled) of variety $i$ in environment $j . \beta_{j}$ is the quadratic effect of HD on GY in environment $j$. All models are summarized in Table 2.

For CVnewE, $\alpha_{j}$ and $\beta_{j}$ cannot be estimated in models 4-9 because there is no observation of $\mathrm{HD}$ and GY in the test environment even for the calibration set, and so an alternative procedure was applied. First, $\alpha_{j}$ and $\beta_{j}$ were estimated using a linear or a quadratic regression of GY on $\mathrm{HD}$ in each environment except the test environment. The estimates of the regression coefficients were then used to fit a multiple linear regression on the EC measured in these environments. A stepwise forward-backward procedure (function "step" of $\mathrm{R}$ package "stats" with a penalty $k=3.7$ for $\alpha$ and $k=8$ for $\beta$ ) was applied to select the most relevant EC and estimate their effects. This calibrated model was then used to predict $\alpha_{j}$ and $\beta_{j}$ in the test environment. The estimates $\hat{\alpha}_{j}$ and $\hat{\beta}_{j}$ were plugged into models 4-9, which could then be used to predict GY in the test environment. We suppose here that the target environments are characterized by EC, so they are not totally unknown.

For each model, predictions were obtained as the sum of the BLUEs of the fixed effects and the BLUPs of the random effects. For each cross-validation scenario, the predictive ability (correlation between the predictions and the adjusted means) and root mean square error (RMSE) of HD in step 1 was estimated, as well as the RMSE of $\alpha_{j}$ and $\beta_{j}$ for CVnewE. The predictive ability of GY in step 2 was evaluated for each model as presented above.

\section{RESULTS}

\begin{tabular}{|c|c|c|c|c|c|}
\hline & \multicolumn{5}{|c|}{ Structure of the models } \\
\hline & $\mathbf{E}$ & $\mathbf{G}$ & GEI & $\mathbf{h}$ & $h^{2}$ \\
\hline \multicolumn{6}{|c|}{ Reference models } \\
\hline$E G$ & $x$ & K & - & - & - \\
\hline EG_GxE & $x$ & K & $K \otimes I_{N_{E}}$ & - & - \\
\hline EG_GxW & $x$ & K & $K \otimes W$ & - & - \\
\hline \multicolumn{6}{|c|}{ CGM-TAP models } \\
\hline EG_HD & $x$ & K & - & $x$ & - \\
\hline EG_GxE_HD & $x$ & K & $K \otimes I_{N_{E}}$ & $x$ & - \\
\hline EG_GxW_HD & $x$ & K & $K \otimes W$ & $x$ & - \\
\hline EG_HD ${ }^{2}$ & $x$ & K & - & $x$ & $x$ \\
\hline EG_GXE_HD ${ }^{2}$ & $x$ & K & $K \otimes I_{N_{E}}$ & $x$ & $\mathrm{x}$ \\
\hline EG_GxW_HD ${ }^{2}$ & $x$ & K & $K \otimes W$ & $x$ & $x$ \\
\hline
\end{tabular}

\section{Relationship Between HD and GY in the Sixteen Environments}

The variance of HD was highly variable from one environment to another (Figure 2). HD was constraint to 15 days in Vra13LN whereas it covered around 30 days in other environments (Gre14WD, Coi12WW).

TABLE 2 | Description of the reference and CGM-TAP models.

It is specified if the effect was included ( $x$ ) or excluded (-) from the model. For mixed effects ( $G$ and GEI) the structure of the covariance matrix is indicated. 


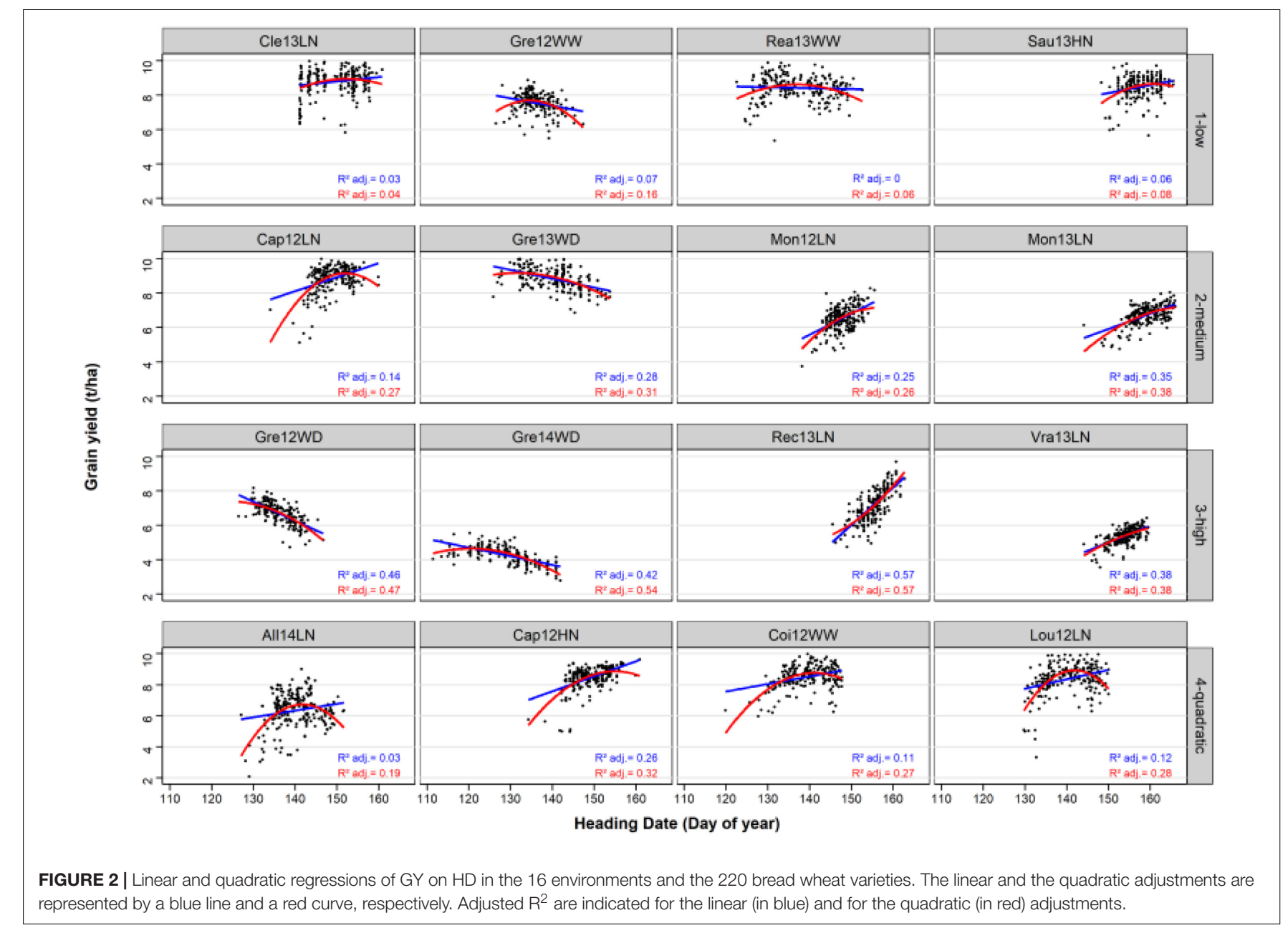

The average adjusted $\mathrm{R}^{2}$ of the linear relationship was of 0.04 , $0.26,0.46$, and 0.13 in the low, medium, high and quadratic classes, respectively. For the quadratic environments, the adjusted $R^{2}$ of the quadratic regression was of 0.27 on average, and higher than the adjusted $R^{2}$ of the linear regression. In all four environments of this class, the quadratic relationship was concave with an optimal HD.

\section{Predictive Ability and RMSE of the Secondary Trait (HD) in the Different Scenarios}

Before looking at the predictive abilities of GY with the different models, we looked at the predictive ability of the secondary trait (HD) in the different cross-validation scenarios (Table 3). The average predictive abilities of $\mathrm{HD}$ were above 0.78 in all scenarios, and it was higher in scenarios CVrandom and CVnewE (0.91) than in CVnewG (0.78). This was expected as the genetic parameters of the CGM had to be predicted in scenario CVnewG. RMSE was low in the three scenarios (average always below 3.33 days), and it was lower for CVrandom (1.86 days) than for CVnewG (2.88 days) and CVnewE (3.33 days). There was a strong variability of RMSE between folds with a maximum of 6.63 days in scenario CVnewE.

\section{Prediction of the Regression Coefficients of HD on GY ( $\alpha$ and $\beta)$ in Scenario CVnewE}

For models EG_HD, EG_GxE_HD, and EG_GxW_HD, it was necessary to first predict the value of $\alpha$ to be able to run the models in CVnewE, because no phenotypic observation is available on the predicted environment and thus, the relationship between HD and GY could not be directly estimated. For the same reasons, both $\alpha$ and $\beta$ should be predicted before running models EG_HD ${ }^{2}$,EG_GxE_HD ${ }^{2}$, and EG_GxW_HD ${ }^{2}$.

The multiple linear regression on the environmental covariates was moderately efficient to predict the value of $\alpha$ for the linear regression of $\mathrm{HD}$ on GY in the new environment (Figure 3A). The RMSE was equal to 0.40 for observed $\alpha$ values varying between -0.99 and 1.91. For all environments except one, the environmental covariates were able to accurately predict the sign of the regression.

The predictions of $\alpha$ and $\beta$ were less accurate for the quadratic linear regression of GY on HD, with RMSE equal to 0.44 for $\alpha$, 
TABLE 3 | Predictive ability and RMSE of the secondary trait (HD) in the three prediction scenarios.

\begin{tabular}{|c|c|c|c|c|c|c|c|c|c|}
\hline & \multicolumn{3}{|c|}{ CVrandom } & \multicolumn{3}{|c|}{ CVnewG } & \multicolumn{3}{|c|}{ CVnewE } \\
\hline & Min. & Mean & Max. & Min. & Mean & Max. & Min. & Mean & Max. \\
\hline Pred. ability & 0.68 & 0.91 & 0.98 & 0.49 & 0.78 & 0.95 & 0.86 & 0.91 & 0.96 \\
\hline RMSE (days) & 0.81 & 1.86 & 3.40 & 1.39 & 2.88 & 5.37 & 1.62 & 3.33 & 6.63 \\
\hline
\end{tabular}

CVrandom corresponds to sparse testing, CVnewG to the prediction of unobserved varieties, and CVnewE to the prediction of unobserved environments. CVrandom and

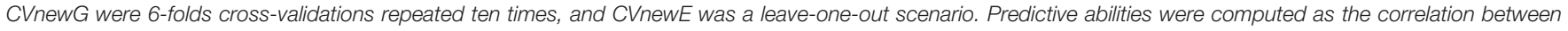
adjusted means and predictions. RMSE are the root mean squared errors.
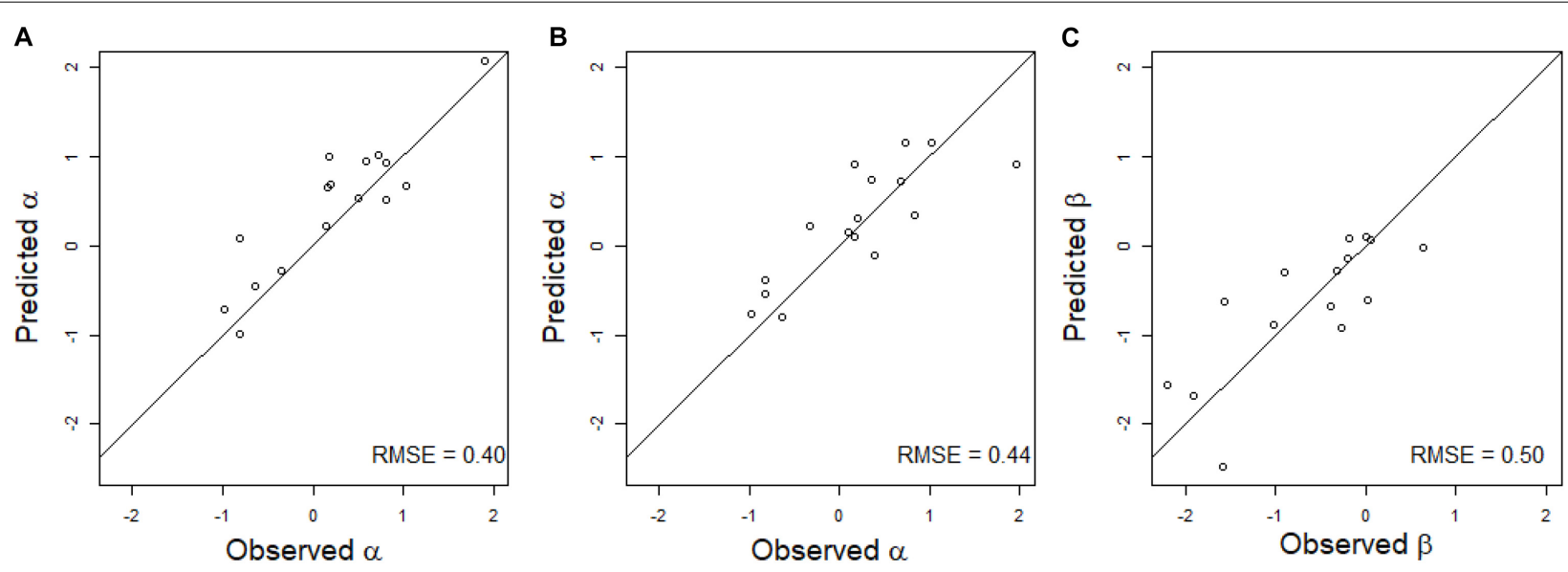

FIGURE 3 | Scatter plot of the predicted and observed values of the regression coefficient of a linear regression (A) or of a quadratic regression (B,C) of GY on HD in scenario CVnewE. Observed $\alpha$ and $\beta$ correspond to the estimates obtained from the linear (A) and quadratic (B,C) regressions of GY on HD in each environment. Predicted $\alpha$ and $\beta$ correspond to the prediction of the $\alpha(\mathbf{A})$ or $\alpha$ and $\beta \mathbf{( B , C )}$ of the predicted environment (leave-one-environment-out scheme), using a multiple linear regression of the $\alpha \mathbf{( A )}$ or $\alpha$ and $\beta \mathbf{( B , C )}$ estimated in the calibration environments on the environmental covariates. The black line corresponds to the line $y=x$.

and 0.50 for $\beta$ (Figures 3B,C). For $\beta$, the sign of the regression was inaccurately predicted for three environments.

\section{Predictive Ability of the Target Trait (GY) in the Different Scenarios}

The predictive abilities for the target trait (GY) were highly variable between scenarios and between models (Table 4). On average over the four kinds of environments (low, medium, high and quadratic), the highest predictive abilities were obtained in scenario CVrandom (0.73 for EG_GxW_HDpred and EG_GxW_HDpred ${ }^{2}$ ). It was slightly lower for CVnewE (0.70 for EG_GxW_HDpred) and CVnewG (0.57 for EG_GxW_HDpred). The inclusion of GEI effects in the model (GxE and GxW) always increased predictive abilities in comparison to the main effect model EG, in particular when EC were used to estimate covariance between environments $(\mathrm{GxW})$. On average over the four kinds of environments, the introduction of term GxW increased predictive abilities by $39 \%$ for scenario CVrandom, $37 \%$ for $\mathrm{CVnewG}$, and $25 \%$ for $\mathrm{CVnewE}$ in comparison to the main effect model.

For all scenarios and kinds of environment, the best model was always a CGM-TAP model, except in CVnewE for "low" and "quadratic" environments. The inclusion of $\mathrm{HD}$ as fixed effect (CGM-TAP) in the three reference models (EG, EG_GxE, and EG_GxW) always increased the average (over the four kinds of environments) predictive abilities. This increase strongly depended on the kind of environment and on the reference model considered. For the environment with a low correlation between GY and HD, and the environments with a quadratic relationship, the inclusion of $\mathrm{HD}$ as fixed effect in the reference models resulted in similar predictive abilities than those of the reference models, or to lower predictive abilities in CVnewE. Inclusion of $\mathrm{HD}$ increased predictive abilities of the reference models for environments with a medium GY/HD relationship. This increase was of 31,30 , and $5 \%$ in CVrandom, CVnewG and CVnewE, respectively, for model EG. As expected, the interest of CGM-TAP was highest for environments with a strong GY/HD relationship ("high"). For these environments the increase brought by CGM-TAP model was of 141, 127, and $132 \%$ in comparison to model EG in CVrandom, CVnewG and CVnewE, respectively. This increase was high but less pronounced for more complex reference models: it was of 7.1, 5.3, and $29.0 \%$ in comparison to model EG_GxW in CVrandom, CVnewG and CVnewE, respectively. Interestingly, in many cases, the simple CGM-TAP models (EG_HDpred, and ED_GxE_HDpred) performed as well as complex model involving environmental covariates (EG_GxW).

The introduction of a quadratic relationship in the reference models only slightly increased predictive abilities in scenario 
TABLE 4 | Predictive abilities of the reference and of the CGM-TAP models in three prediction scenarios with HD as the secondary trait and GY as the target trait.

\begin{tabular}{|c|c|c|c|c|c|c|c|c|c|c|c|c|c|c|c|}
\hline \multirow[b]{2}{*}{ Models } & \multicolumn{5}{|c|}{ CVrandom } & \multicolumn{5}{|c|}{ CVnewG } & \multicolumn{5}{|c|}{ CVnewE } \\
\hline & Low & Medium & High & Quadratic & Average & Low & Medium & High & Quadratic & Average & Low & Medium & High & Quadratic & Average \\
\hline$E G$ & 0.57 & 0.55 & 0.27 & 0.66 & 0.51 & 0.44 & 0.43 & 0.22 & 0.52 & 0.40 & 0.61 & 0.57 & 0.28 & 0.68 & 0.54 \\
\hline EG_HDpred & 0.59 & 0.72 & 0.65 & 0.66 & 0.66 & 0.46 & 0.56 & 0.50 & 0.53 & 0.51 & 0.65 & 0.60 & 0.66 & 0.64 & 0.64 \\
\hline EG_HD ${ }^{2}$ pred & 0.59 & 0.71 & 0.66 & 0.68 & 0.66 & 0.41 & 0.55 & 0.48 & 0.40 & 0.46 & 0.64 & 0.62 & 0.66 & 0.14 & 0.51 \\
\hline EG_GxE & 0.66 & 0.67 & 0.54 & 0.67 & 0.64 & 0.53 & 0.57 & 0.56 & 0.54 & 0.55 & - & - & - & - & - \\
\hline EG_GxE_HDpred & 0.67 & 0.73 & 0.65 & 0.68 & 0.68 & 0.54 & 0.59 & 0.59 & 0.54 & 0.57 & - & - & - & - & - \\
\hline EG_GxE_HD ${ }^{2}$ pred & 0.66 & 0.71 & 0.64 & 0.70 & 0.68 & 0.50 & 0.59 & 0.54 & 0.45 & 0.52 & - & - & - & - & - \\
\hline EG_GxW & 0.71 & 0.73 & 0.70 & 0.70 & 0.71 & 0.54 & 0.57 & 0.57 & 0.53 & 0.55 & 0.68 & 0.69 & 0.62 & 0.70 & 0.67 \\
\hline EG_GxW_HDpred & 0.72 & 0.76 & 0.75 & 0.70 & 0.73 & 0.55 & 0.59 & 0.60 & 0.54 & 0.57 & 0.65 & 0.68 & 0.80 & 0.66 & 0.70 \\
\hline EG_GxW_HD ${ }^{2}$ pred & 0.71 & 0.75 & 0.74 & 0.72 & 0.73 & 0.52 & 0.59 & 0.55 & 0.46 & 0.53 & 0.60 & 0.73 & 0.78 & 0.24 & 0.59 \\
\hline
\end{tabular}

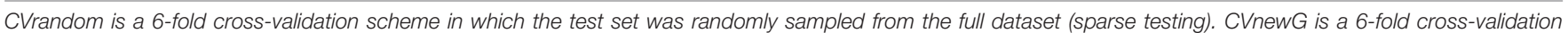

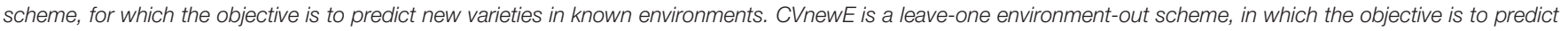

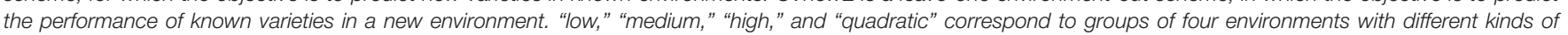

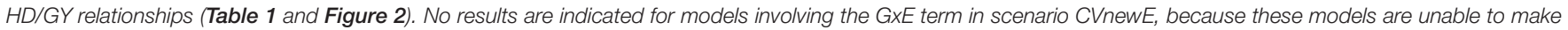
predictions in environments without any phenotypic data.

CVrandom. In other scenarios, the introduction of a quadratic relationship resulted in similar or lower abilities. It considerably decreased predictive abilities in scenario CVnewE, in which the regression coefficients ( $\alpha$ and $\beta$ ) have to be predicted first using the EC (there is no phenotypic information in the test environments, and so the regression coefficients of the GY/HD relationship cannot be directly estimated).

\section{DISCUSSION}

In plant breeding, the knowledge that we have on the candidate varieties is limited because of the necessity to control phenotyping costs and the duration of the breeding cycles. This lack of information considerably limits the chance of identifying top varieties candidate for the registration process. Genomic prediction is a promising tool to screen early generation material (Heffner et al., 2010), because it allows transferring the information collected on the previous breeding cycles to other combination of genes and environmental conditions even in presence of GEI. Different models were proposed in the past years to predict GEI, which involved genetic and environmental characteristics (markers and EC) (Heslot et al., 2014; Jarquín et al., 2014; Cooper et al., 2016; Ly et al., 2018). We developed a new approach, called CGM-TAP, in which a secondary trait is predicted for the test set in each environment thanks to CGM. This secondary trait, easy to predict and capturing GEI, is then used as an environment specific covariate in usual GEI GS models. To sum up, it is a trait assisted prediction model, in which the secondary trait is predicted thanks to CGM instead of being phenotyped. Here we used HD and GY as secondary and target traits, respectively, to evaluate this new approach.

The results presented here reveal that in almost all situations, the CGM-TAP models performed at least as good as the corresponding reference models. It performed even better than the most sophisticated reference model involving EC (EG_GxW) with an increase of predictive ability of $7.1,5.3$, and $29.0 \%$ for the environments with the highest correlation between HD and GY ("high") in scenarios CVrandom, CVnewG and CVnewE, respectively. The increase of predictive ability was generally stronger when the relationship between the secondary and the target trait was strong, as expected. But even for the "low" environments, CGM-TAP models (without the quadratic relationship) performed at least as good as the reference models. In the three cross-validation scenarios there were even situations in which the simple CGM-TAP model including only the main effects and the secondary trait (EG_HD) performed as good as the most sophisticated reference model EG_GxW (Table 4).

However, the introduction of a quadratic effect in the CGMTAP models did not lead to a higher predictive ability and was even detrimental for the "quadratic" environments in scenario CVnewE. This is because we were unable to predict the regression coefficients $\alpha$ and $\beta$ sufficiently well in new environments (Figure 3). The use of a larger set of environments would probably help to better predict these coefficients. Another option would be to use CGM to directly predict the HD/GY relationship in new environments, but we were unable to do it accurately. In other prediction scenarios (CVrandom and CVnewG), the introduction of the quadratic effect resulted in similar or lower predictive abilities in comparison to the CGM-TAP models without quadratic effect. This may be due to the necessity to reach more precise prediction of the secondary trait if a quadratic relationship is fitted. Another explanation is that the quadratic relationships were not very pronounced in this dataset (Figure 2).

These promising results show that the predictions of the secondary trait by the CGM were sufficiently accurate to be able to run a trait-assisted prediction model (without quadratic relationship) in all three prediction scenarios without phenotyping the test set. The RMSE of the prediction of HD with the CGM were indeed relatively low (Table 3). It was equal to 1.86 days in scenario CVrandom, which is close to usual field phenotyping error for this trait. The interest of the CGM-TAP models is dependent on our ability to predict the secondary trait. 
This means that CGM-TAP should work better if the calibration set was bigger, or was phenotyped in a MET optimized for the genetic parameters estimation (Rincent et al., 2017). Note that the use of the observed HD instead of the predicted HD as covariate in the CGM-TAP models resulted in much higher predictive abilities (results no shown). This means that CGM-TAP could work much better if we were able to predict HD more accurately.

Most studies on multi-trait and trait-assisted predictions are based on mixed models involving a genetic correlation between secondary and target traits (Rutkoski et al., 2016; Sun et al., 2017; Michel et al., 2018; Lado et al., 2018; Schulthess et al., 2018). Here, we proposed to introduce the secondary trait as covariate as in Crain et al. (2018), which allows modeling non-linear relationship between traits. This is useful in some situations with complex dependencies as illustrated by the quadratic relationship between HD and GY in some environments. This could also be the case with other secondary traits, for which there is an optimum value depending on the environment such as aerial or root biomass.

The choice of $\mathrm{HD}$ as a secondary trait was justified by the fact that it is easily predicted by CGM (Bogard et al., 2014), and it is an adaptive trait, and so is expected to be related to GY, at least in some environments (Richards, 1991; Semenov et al., 2014; Flohr et al., 2017). The relationship between phenology and productivity is important for most species (Andrade, 1995; Ouk et al., 2007), and so we can expect our strategy to be valuable for other crops. This relationship was confirmed in our dataset in which environments with strong HD/GY correlations or quadratic relationship could be found (Figure 2). However, the range of $\mathrm{HD}$ in breeding programs would be narrower and the correlation probably less pronounced. HD was a good candidate to test this approach, but other traits even more related to the target trait could be used. The important development of highthroughput phenotyping and of crop-growth modeling should make it possible to predict other yield related traits in the future. A suitable secondary trait to run CGM-TAP models should be accurately predicted with CGM and strongly related to the target trait. Traits related to light interception (LAI) or even yield components are secondary traits of choice and may be accurately predicted thanks to CGM in the near future with the help of HTP.

Note that the predictive abilities obtained in scenario CVnewG are probably higher in this study as would be in practice, as the folds of the cross-validation were randomly sampled. This indeed implies that varieties in the calibration set and in the test set are sampled from a same population, which is not the case in a real breeding scheme. Considering the reference models, the results of this study confirm that the introduction of an interaction (GEI) term in the prediction model increased predictive abilities in comparison to a main effect model for all scenarios. The model involving the environmental covariance matrix W (EG_GxW), performed better than model EG_GxE in scenario CVrandom, but similarly in scenario CVnewG. A major advantage of EG_GxW is that it allowed predicting in new environments, and these predictions were much more accurate than the main effect model. This is in agreement with previous publications (Jarquín et al., 2014; Malosetti et al., 2016; Rincent et al., 2019).

\section{CONCLUSION}

In this paper, we propose a new way to predict genotype by environment interactions (CGM-TAP). This is a trait-assisted prediction approach in which the secondary trait is predicted by a crop growth model, instead of being phenotyped. The relationship between the secondary trait and the target trait is environment specific and thus allow predicting environment specific effects. This approach applied to yield (target trait) and heading date (secondary trait) increased predictive abilities of all reference GEI models, even those involving sophisticated environmental covariates, for various prediction scenarios (new varieties, new environments and sparse testing). This proof of concept could be applied to other traits in breeding programs in the near future, and is a new way of combining crop growth modeling and genomic prediction in the context of GEI.

\section{DATA AVAILABILITY STATEMENT}

The data supporting the conclusions of this article can be found at https://doi.org/10.15454/TKMGCQ.

\section{AUTHOR CONTRIBUTIONS}

RR defined the prediction strategy and prediction models, ran the analysis, and wrote the manuscript. PR ran the analysis and reviewed the manuscript. JL reviewed the manuscript, participated in the discussions, and contributed to the data production with the BreedWheat Consortium. All authors contributed to the article and approved the submitted version.

\section{FUNDING}

Management of the wheat multi-environment trials was financially supported by the French National Research National Agency under Investment for the Future (BreedWheat project ANR-10-BTBR-03) and by FranceAgriMer. The Phéno3C platform was financially funded by the French National Research National Agency under the Investment for the Future Phenome project (ANR-11-INBS-12) and by the European Regional Development Fund (AV0011535). We thank H2020 INVITE for financial support (grant agreement no. 817970).

\section{ACKNOWLEDGMENTS}

We thank three reviewers for their relevant comments which improved the manuscript.

\section{SUPPLEMENTARY MATERIAL}

The Supplementary Material for this article can be found online at: https://www.frontiersin.org/articles/10.3389/fpls.2020.00827/ full\#supplementary-material 


\section{REFERENCES}

Andrade, F. H. (1995). Analysis of growth and yield of maize, sunflower and soybean grown at Balcarce, Argentina. Field Crops Res. 41, 1-12. doi: 10.1016/ 0378-4290(94)00107-N

Bogard, M., Ravel, C., Paux, E., Bordes, J., Balfourier, F., Chapman, S. C., et al. (2014). Predictions of heading date in bread wheat (Triticum aestivum L.) using QTL-based parameters of an ecophysiological model. J. Exp. Bot. 65, 5849-5865. doi: 10.1093/jxb/eru328

Burgueño, J., de los Campos, G., Weigel, K., and Crossa, J. (2012). Genomic prediction of breeding values when modeling genotype $\times$ environment interaction using pedigree and dense molecular markers. Crop Sci. 52, 707-719. doi: $10.2135 /$ cropsci2011.06.0299

Bustos-Korts, D., Boer, M. P., Malosetti, M., Chapman, S., Chenu, K., Zheng, B., et al. (2019). Combining crop growth modeling and statistical genetic modeling to evaluate phenotyping strategies. Front. Plant Sci. 10:1491. doi: 10.3389/fpls. 2019.01491

Calus, M. P., and Veerkamp, R. F. (2011). Accuracy of multi-trait genomic selection using different methods. Genet. Sel. Evol. 43:26. doi: 10.1186/1297-968643-26

Charmet, G., Tran, L.-G., Auzanneau, J., Rincent, R., and Bouchet, S. (2020). BWGS: a $\mathrm{R}$ package for genomic selection and its application to a wheat breeding programme. PLoS One 15:e0232422. doi: 10.1371/journal.pone. 0232422

Chenu, K., Chapman, S. C., Hammer, G. L., Mclean, G., Salah, H. B. H., and Tardieu, F. (2008). Short-term responses of leaf growth rate to water deficit scale up to whole-plant and crop levels: an integrated modelling approach in maize. Plant Cell Environ. 31, 378-391. doi: 10.1111/j.1365-3040.2007.01772.x

Cooper, M., Technow, F., Messina, C., Gho, C., and Totir, L. R. (2016). Use of crop growth models with whole-genome prediction: application to a maize multienvironment trial. Crop Sci. 56, 2141-2156. doi: 10.2135/cropsci2015.08. 0512

Crain, J., Mondal, S., Rutkoski, J., Singh, R. P., and Poland, J. (2018). Combining high-throughput phenotyping and genomic information to increase prediction and selection accuracy in wheat breeding. Plant Genome 11:UNS170043. doi: 10.3835/plantgenome2017.05.0043

Crossa, J., de los Campos, G., Maccaferri, M., Tuberosa, R., Burgueño, J., and Pérez-Rodríguez, P. (2016). Extending the marker $\times$ environment interaction model for genomic-enabled prediction and genome-wide association analysis in durum wheat. Crop Sci. 56, 2193-2209. doi: 10.2135/cropsci2015.04.0260

Damesa, T., Worku, M., Möhring, J., and Piepho, H. P. (2017). One step at a time: stage-wise analysis of a series of experiments. Agron. J. 109, 845-857. doi: 10.2134/agronj2016.07.0395

Fernandes, S. B., Dias, K. O. G., Ferreira, D. F., and Brown, P. J. (2018). Efficiency of multi-trait, indirect, and trait-assisted genomic selection for improvement of biomass sorghum. Theor. Appl. Genet. 131, 747-755. doi: 10.1007/s00122-0173033-y

Flohr, B. M., Hunt, J. R., Kirkegaard, J. A., and Evans, J. R. (2017). Water and temperature stress define the optimal flowering period for wheat in southeastern Australia. Field Crops Res. 209, 108-119. doi: 10.1016/j.fcr.2017.04.012

Heffner, E. L., Lorenz, A. J., Jannink, J. L., and Sorrells, M. E. (2010). Plant breeding with genomic selection: gain per unit time and cost. Crop Sci. 50, 1681-1690. doi: $10.2135 /$ cropsci2009.11.0662

Henderson, C., and Quaas, R. (1976). Multi-trait selection using relatives records. J. Anim. Sci. 43, 218-218.

Heslot, N., Akdemir, D., Sorrells, M. E., and Jannink, J.-L. (2014). Integrating environmental covariates and crop modeling into the genomic selection framework to predict genotype by environment interactions. Theor. Appl. Genet. 127, 463-480. doi: 10.1007/s00122-013-2231-5

Jarquín, D., Crossa, J., Lacaze, X., Du Cheyron, P., Daucourt, J., Lorgeou, J., et al. (2014). A reaction norm model for genomic selection using high-dimensional genomic and environmental data. Theor. Appl. Genet. 127, 595-607. doi: 10. 1007/s00122-013-2243-1

Jia, Y., and Jannink, J.-L. (2012). Multiple-trait genomic selection methods increase genetic value prediction accuracy. Genetics 192, 1513-1522. doi: 10.1534/ genetics.112.144246

Lado, B., Vazquez, D., Quincke, M., Silva, P., Aguilar, I., and Gutierrez, L. (2018). Resource allocation optimization with multi-trait genomic prediction for bread wheat (Triticum aestivum L.) baking quality. Theor. Appl. Genet. 131, 27192731. doi: 10.1007/s00122-018-3186-3

Lopes, M. S., Saglam, D., Ozdogan, M., and Reynolds, M. (2014). Traits associated with winter wheat grain yield in Central and West Asia. J. Integr. Plant Biol. 56, 673-683. doi: 10.1111/jipb.12172

Ly, D., Chenu, K., Gauffreteau, A., Rincent, R., Huet, S., Gouache, D., et al. (2017). Nitrogen nutrition index predicted by a crop model improves the genomic prediction of grain number for a bread wheat core collection. Field Crops Res. 214, 331-340. doi: 10.1016/j.fcr.2017.09.024

Ly, D., Huet, S., Gauffreteau, A., Rincent, R., Touzy, G., Mini, A., et al. (2018). Whole-genome prediction of reaction norms to environmental stress in bread wheat (Triticum aestivum L.) by genomic random regression. Field Crops Res. 216, 32-41. doi: 10.1016/j.fcr.2017.08.020

Malosetti, M., Bustos-Korts, D., Boer, M. P., and van Eeuwijk, F. A. (2016). Predicting responses in multiple environments: issues in relation to genotype $\times$ environment interactions. Crop Sci. 56, 2210-2222. doi: 10.2135/cropsci2015. 05.0311

Martre, P., Jamieson, P. D., Semenov, M. A., Zyskowski, R. F., Porter, J. R., and Triboi, E. (2006). Modelling protein content and composition in relation to crop nitrogen dynamics for wheat. Eur. J. Agron. 25, 138-154. doi: 10.1016/j. eja.2006.04.007

Messina, C. D., Jones, J. W., Boote, K. J., and Vallejos, C. E. (2006). A gene-based model to simulate soybean development and yield responses to environment. Crop Sci. 46, 456-466. doi: 10.2135/cropsci2005.04-0372

Messina, C. D., Technow, F., Tang, T., Totir, R., Gho, C., and Cooper, M. (2018). Leveraging biological insight and environmental variation to improve phenotypic prediction: integrating crop growth models (CGM) with whole genome prediction (WGP). Eur. J. Agron. 100, 151-162. doi: 10.1016/j.eja.2018. 01.007

Meuwissen, T. H., Hayes, B. J., and Goddard, M. E. (2001). Prediction of total genetic value using genome-wide dense marker maps. Genetics 157, 1819-1829.

Michel, S., Kummer, C., Gallee, M., Hellinger, J., Ametz, C., Akgöl, B., et al. (2018). Improving the baking quality of bread wheat by genomic selection in early generations. Theor. Appl. Genet. 131, 477-493. doi: 10.1007/s00122-0172998-x

Nakagawa, H., Yamagishi, J., Miyamoto, N., Motoyama, M., Yano, M., and Nemoto, K. (2005). Flowering response of rice to photoperiod and temperature: a QTL analysis using a phenological model. Theor. Appl. Genet. 110, 778-786. doi: 10.1007/s00122-004-1905-4

Onogi, A., Watanabe, M., Mochizuki, T., Hayashi, T., Nakagawa, H., Hasegawa, T., et al. (2016). Toward integration of genomic selection with crop modelling: the development of an integrated approach to predicting rice heading dates. Theor. Appl. Genet. 129, 805-817. doi: 10.1007/s00122-016-2667-5

Ouk, M., Basnayake, J., Tsubo, M., Fukai, S., Fischer, K. S., Kang, S., et al. (2007). Genotype-by-environment interactions for grain yield associated with water availability at flowering in rainfed lowland rice. Field Crops Res. 101, 145-154. doi: $10.1016 /$ j.fcr.2006.10.003

Prudent, M., Lecomte, A., Bouchet, J.-P., Bertin, N., Causse, M., and Genard, M. (2011). Combining ecophysiological modelling and quantitative trait locus analysis to identify key elementary processes underlying tomato fruit sugar concentration. J. Exp. Bot. 62, 907-919. doi: 10.1093/jxb/erq318

Pszczola, M., Veerkamp, R. F., de Haas, Y., Wall, E., Strabel, T., and Calus, M. P. L. (2013). Effect of predictor traits on accuracy of genomic breeding values for feed intake based on a limited cow reference population. Animal 7, 1759-1768. doi: 10.1017/S175173111300150X

Purcell, S., Neale, B., Todd-Brown, K., Thomas, L., Ferreira, M. A. R., Bender, D., et al. (2007). PLINK: a tool set for whole-genome association and populationbased linkage analyses. Am. J. Hum. Genet. 81, 559-575. doi: 10.1086/5 19795

Quilot, B., Génard, M., Lescourret, F., and Kervella, J. (2005). Simulating genotypic variation of fruit quality in an advanced peach $\times$ Prunus davidiana cross. J. Exp. Bot. 56, 3071-3081. doi: 10.1093/jxb/eri304

Reymond, M., Muller, B., Leonardi, A., Charcosset, A., and Tardieu, F. (2003). Combining quantitative trait loci analysis and an ecophysiological model to analyze the genetic variability of the responses of maize leaf growth to temperature and water deficit. Plant Physiol. 131, 664-675. doi: 10.1104/pp. 013839 
Reymond, M., Muller, B., and Tardieu, F. (2004). Dealing with the genotype x environment interaction via a modelling approach: a comparison of QTLs of maize leaf length or width with QTLs of model parameters. J. Exp. Bot. 55, 2461-2472. doi: 10.1093/jxb/erh200

Richards, R. (1991). Crop improvement for temperate Australia - future opportunities. Field Crops Res. 26, 141-169. doi: 10.1016/0378-4290(91)90033$\mathrm{R}$

Rimbert, H., Darrier, B., Navarro, J., Kitt, J., Choulet, F., Leveugle, M., et al. (2018). High throughput SNP discovery and genotyping in hexaploid wheat. PLoS One 13:e0186329. doi: 10.1371/journal.pone.0186329

Rincent, R., Charpentier, J.-P., Faivre-Rampant, P., Paux, E., Le Gouis, J., Bastien, C., et al. (2018). Phenomic selection is a low-cost and high-throughput method based on indirect predictions: proof of concept on wheat and poplar. G3 (Bethesda) 8, 3961-3972. doi: 10.1534/g3.118.200760

Rincent, R., Kuhn, E., Monod, H., Oury, F.-X., Rousset, M., Allard, V., et al. (2017). Optimization of multi-environment trials for genomic selection based on crop models. Theor. Appl. Genet. 130, 1735-1752. doi: 10.1007/s00122017-2922-4

Rincent, R., Malosetti, M., Ababaei, B., Touzy, G., Mini, A., Bogard, M., et al. (2019). Using crop growth model stress covariates and AMMI decomposition to better predict genotype-by-environment interactions. Theor. Appl. Genet. 132, 3399-3411. doi: 10.1007/s00122-019-03432-y

Rodríguez-Álvarez, M. X., Boer, M. P., van Eeuwijk, F. A., and Eilers, P. H. C. (2018). Correcting for spatial heterogeneity in plant breeding experiments with P-splines. Spat. Stat. 23, 52-71. doi: 10.1016/j.spasta.2017.10.003

Rutkoski, J., Poland, J., Mondal, S., Autrique, E., Pérez, L. G., Crossa, J., et al. (2016). Canopy temperature and vegetation indices from highthroughput phenotyping improve accuracy of pedigree and genomic selection for grain yield in wheat. G3 (Bethesda) 6, 2799-2808. doi: 10.1534/g3.116. 032888

Schulthess, A. W., Zhao, Y., Longin, C. F. H., and Reif, J. C. (2018). Advantages and limitations of multiple-trait genomic prediction for Fusarium head blight severity in hybrid wheat (Triticum aestivum L.). Theor. Appl. Genet. 131, 685-701. doi: 10.1007/s00122-017-3029-7

Schulz-Streeck, T., Ogutu, J. O., Gordillo, A., Karaman, Z., Knaak, C., and Piepho, H.-P. (2013). Genomic selection allowing for marker-by-environment interaction. Plant Breed. 132, 532-538. doi: 10.1111/pbr.12105

Semenov, M. A., Stratonovitch, P., Alghabari, F., and Gooding, M. J. (2014). Adapting wheat in Europe for climate change. J. Cereal Sci. 59, 245-256. doi: 10.1016/j.jcs.2014.01.006

Sun, J., Poland, J. A., Mondal, S., Crossa, J., Juliana, P., Singh, R. P., et al. (2019). High-throughput phenotyping platforms enhance genomic selection for wheat grain yield across populations and cycles in early stage. Theor. Appl. Genet. 132, 1705-1720. doi: 10.1007/s00122-019-03309-0
Sun, J., Rutkoski, J. E., Poland, J. A., Crossa, J., Jannink, J.-L., and Sorrells, M. E. (2017). Multitrait, random regression, or simple repeatability model in highthroughput phenotyping data improve genomic prediction for wheat grain yield. Plant Genome 10:28724067. doi: 10.3835/plantgenome2016.11.0111

Technow, F., Messina, C. D., Totir, L. R., and Cooper, M. (2015). Integrating crop growth models with whole genome prediction through approximate bayesian computation. PLoS One 10:e0130855. doi: 10.1371/journal.pone.0130855

Touzy, G., Rincent, R., Bogard, M., Lafarge, S., Dubreuil, P., Mini, A., et al. (2019). Using environmental clustering to identify specific drought tolerance QTLs in bread wheat (T. aestivum L.). Theor. Appl. Genet. 132, 2859-2880. doi: 10.1007/s00122-019-03393-2

Uptmoor, R., Li, J., Schrag, T., and Stützel, H. (2011). Prediction of flowering time in Brassica oleracea using a quantitative trait loci-based phenology model: flowering time in Brassica oleracea. Plant Biol. 14, 179-189. doi: 10.1111/j.14388677.2011.00478.x

VanRaden, P. M. (2008). Efficient methods to compute genomic predictions. J. Dairy Sci. 91, 4414-4423. doi: 10.3168/jds.2007-0980

White, J. W., Herndl, M., Hunt, L. A., Payne, T. S., and Hoogenboom, G. (2008). Simulation-based analysis of effects of Vrn and Ppd loci on flowering in wheat. Crop Sci. 48, 678-687. doi: 10.2135/cropsci2007.06.0318

White, J. W., and Hoogenboom, G. (1996). Simulating effects of genes for physiological traits in a process-oriented crop model. Agron. J. 88, 416-422. doi: 10.2134/agronj1996.00021962008800030009x

Whittaker, J. C., Thompson, R., and Denham, M. C. (2000). Marker-assisted selection using ridge regression. Genet. Res. 75, 249-252. doi: 10.1017/ S0016672399004462

Yin, X. (2005). QTL analysis and QTL-based prediction of flowering phenology in recombinant inbred lines of barley. J. Exp. Bot. 56, 967-976. doi: 10.1093/jxb/ eri090

Zheng, B., Biddulph, B., Li, D., Kuchel, H., and Chapman, S. (2013). Quantification of the effects of VRN1 and Ppd-D1 to predict spring wheat (Triticum aestivum) heading time across diverse environments. J. Exp. Bot. 64, 3747-3761. doi: $10.1093 /$ jxb/ert209

Conflict of Interest: The authors declare that the research was conducted in the absence of any commercial or financial relationships that could be construed as a potential conflict of interest.

Copyright (c) 2020 Robert, Le Gouis and Rincent. This is an open-access article distributed under the terms of the Creative Commons Attribution License (CC BY). The use, distribution or reproduction in other forums is permitted, provided the original author(s) and the copyright owner(s) are credited and that the original publication in this journal is cited, in accordance with accepted academic practice. No use, distribution or reproduction is permitted which does not comply with these terms. 